\title{
PODE O MELHORAMENTO HUMANO SER ACEITO COMO UM DOS OBJETIVOS DA MEDICINA?
}

\author{
Marco Antonio Azevedo \\ Universidade do Vale do Rio dos Sinos - Unisinos - Brasil
}

\section{Resumo:}

Seria razoável e eticamente permissível incluir melhoramentos, ao lado da prevenção e dos tratamentos, como objetivos da medicina? Segundo alguns críticos isso seria inaceitável. Uma das razões é que, se um medicamento ou técnica não protege, promove ou recupera a saúde, na ignorância, deve-se evitá-lo, já que não se pode excluir de antemão a possibilidade de efeitos indesejáveis. Nessa ótica, melhoramentos deveriam ser contraindicados, já que seriam potencialmente iatrogênicos. Porém, e se houver evidências suficientes de que não há parefeitos significativos? Nesse caso, não deveriam os médicos indicá-los? O problema é que se um medicamento for comprovadamente benéfico para o tratamento ou alívios dos sintomas de uma doença, não prescrevê-lo implica negligência. Suponhamos que aceitemos um dia a inclusão de práticas melhoristas no rol dos objetivos da medicina. Se um médico não recomendar um melhoramento, estaria ele também agindo em negligência? Teriam os pacientes direitos sobre seus médicos não só ao melhor tratamento mas também às melhores opções de melhoramento humano? Neste artigo, pretendo tratar desse tema recente de forma exploratória. Pretendo defender que a aceitação dos melhoramentos no âmbito da medicina não implica tomá-los como equivalentes às ações preventivas ou terapêuticas, e que a permissão de melhoramentos não acarreta obrigações sobre os médicos nem direitos a seus pacientes.

Palavras-chave: Melhoramento Humano, Tratamentos e Melhoramentos, Fins da Medicina, Ética Profissional, Ética Médica, Bioética.

\section{A nova era dos melhoramentos e a prática da medicina}

O tema do melhoramento humano (em inglês, human enhancement) é recente em bioética e em filosofia da medicina. Por outro lado, o desejo de superar nossas limitações biológicas é certamente antigo. Ele parece estar ligado à história da técnica e de suas diferentes possibilidades de emprego. O temor diante das possibilidades de uso da técnica também é antigo (lembremo-nos aqui do repetidamente revisitado mito de Prometeu acorrentado). Mas dúvidas filosóficas tendo em vista a possível dissociação entre os fins visados pela prática médica tradicional e os fins "melhoristas", visados especialmente pelas novas biotecnologias aplicadas à medicina, são mais recentes. 
O uso de tecnologias médicas visando produzir estados melhorados e não meramente "saudáveis" tomou um rumo realista a partir do uso disseminado de medicamentos, originalmente criados com a finalidade de modular estados patológicos de humor com a finalidade de melhorar a atenção e o desempenho cognitivo. Em meados da década de 70, a empresa farmacêutica Elli Lilly desenvolveu o novo antidepressivo, a fluoxetina, um inibidor seletivo da recaptação da serotonina (Elliot 2003). Uma das vantagens da fluoxetina sobre os antidepressivos tradicionais é seu menor índice de parefeitos. Mas a possibilidade de que variações fisiológicas, comuns e não patológicas do humor também pudessem ser manejadas sem prejuízos ou parefeitos significativos levou muitos usuários a utilizar o "Prosac" (nome comercial do fármaco fluoxetina fabricado originalmente pela empresa farmacêutica Elli Lilly) não como andidepressivo ou como modulador do humor, mas como estimulante ou euforizante leve. Ora, nessa forma de emprego, a indicação do medicamento não visa a tratar qualquer doença ou desordem mental. O objetivo é claramente melhorar o humor e a disposição mental para além dos padrões do que em medicina se entende como sendo normal.

Algo semelhante veio a ocorrer com o metilfenidato (comercialmente conhecido pelo nome de "Ritalina"). O metilfenidato está indicado para o tratamento da Perturbação por Deficit de Atenção (PDA) (Scheffler 2009, Rapoport \& Inoff-Germain 2002) ao passo que o modafinil está indicado para o tratamento da condição conhecida como Narcolepsia (Kumar 2008). Quanto ao metilfenidato, sabe-se que foi sintetizado em 1944, originalmente com a finalidade de controlar o fenômeno fisiológico da Hipotensão Ortostática (vulgarmente, “Pressão Baixa”) (Elliot 2003). Após a década de 60, o medicamento passou a ser utilizado também para o controle da PDA (Rapoport et al 1980). Como se trata de uma substância capaz de melhorar a atenção e a memória mesmo em pessoas sem PDA, o medicamento começou também a ser usado como estimulante cognitivo. Na década de 80, alguns estudos passaram a mostrar que em baixas doses esses medicamentos neuroestimulantes, incluindo especialmente, o metilfenidado e a dextroanfetamina, são capazes de promover melhoramento cognitivo também em pessoas saudáveis (Rapoport et al 1980, Spencer, Devilbiss \& Berridge 2015, Battleday \& Brem 2015).

O Prozac e a Ritalina ensejam casos típicos de uso de substâncias originalmente desenvolvidas para fins médicos, mas imediatamente empregadas em situações "nãomédicas". Isso levou vários especialistas a recomendar um controle mais rígido da sua prescrição. Contudo, muito embora se tratem de medicamentos prescritos, no Brasil, exclusivamente por receituário médico especial, ambos vêm sendo receitados para situações que não ensejam transtornos clínicos ou patologias. A fluoxetina, por exemplo, vem sendo

Barbarói, Santa Cruz do Sul, Edição Especial n.44, p.<290-303>, jul./dez. 2015 
largamente usada não só para o controle de variações fisiológicas do humor, mas também com a intenção de promover ou aumentar a sensação subjetiva de autoconfiança, em que pese as reiteradas precauções quanto a sua não indicação para indivíduos saudáveis (Marken \& Munro 2000, p. 206). Com o surgimento recente de novos ISRS (inibidores seletivos da recaptação da serotonina), a fluoxetina deixou de ser um medicamento de primeira escolha no tratamento da depressão. Quanto ao metilfenitado, pacientes passaram a demandar dos médicos a sua prescrição como psicoestimulante, especialmente para situações em que se deseja um incremento no desempenho cognitivo. Alguns psicoestimulantes mais novos, como o modafinil, já vem sendo empregados, embora sem prescrição, com a finalidade de aumentar o desempenho cognitivo e aumentar a tolerância ao sono em sujeitos saudáveis. De todo modo, em nenhum desses casos, o uso segue as indicações tidas como especificamente médicas.

\section{Estaria a medicina hipocrática em franco declínio?}

É possível que a emergência dessas novas práticas seja resultado da adaptação do uso da tecnologia médica às recentes demandas dos pacientes por um novo tipo de atenção ou cuidado. O fenômeno parece não estar dissociado da emergência da demanda dos pacientes pelo respeito a seus desejos como consumidores. A emergência da condição dos pacientes como consumidores envolvidos em relações de mercado é um tema bem estudado em Bioética. Tratei disso em meu livro Bioética Fundamental (2002) e, antes disso, no artigo "A profissão sob risco" (1988). Naquela ocasião, chamavam-me a atenção argumentos que poderíamos chamar de "conservadores", os quais diagnosticavam corretamente a existência de pelo menos dois modelos distintos de prática da medicina, o modelo da medicina entendida como mera técnica (à disposição, de forma não moralmente constrangida, dos desejos e interesses variáveis de seus consumidores), e o da medicina como profissão tradicional (isto é, como uma ocupação guiada por uma tradição e protegida legalmente por uma comunidade de praticantes—que também servem-se dela como meio e modo de viver a vida).

Dentre os que entendem a medicina como paradigma de profissão tradicional está o sociólogo Eliot Freidson (1988). Segundo Freidson, profissões são ocupações que obtiveram a permissão legal de exercer o monopólio sobre certa domínnio de atividade técnica, passando a ocupar uma posição de preeminência sobre as opiniões leigas (Freidson 1988, 2001). Partindo dessa ideia, há quem vá ainda mais longe, argumentando em favor de que haveria motivos para se proteger a integridade da tradição hipocrática, reagindo contra as mudanças 
recomendadas pela nova bioética. Incorporar mudanças na tradição (como a aceitação das práticas do aborto e da eutanásia, por exemplo) implicaria, para esse pensamento conservador, desconstituir por completo a integridade da tradição representada pelo modelo hipocrático (que tradicionalmente condena qualquer forma de interrupção ativa mediante ação comissiva ou omitiva) (Cameron 1991). Para Nigel Cameron, o modelo hipocrático possui uma tecitura coesa que não admite remendos ou costuras (a seamless dress); para ele, não há como alterála sem destruí-la por compelto. Daí sua aversão à legalização de práticas tradicionalmente proscritas pelo modelo hipocrático, como a permissão do aborto e a licença moral à eutanásia.

$\mathrm{O}$ argumento de Cameron não é, contudo, persuasivo. A atitude conservadora diante das mudanças da ética profissional médica é, além de ingênua, é incorreta. Afinal, tradições mudam com o tempo. Alasdair MacIntyre, um dos defensores do papel das narrativas tradicionais no desenvolvimento da cultura, foi um dos que destacaram o fato inequívoco de que as tradições evoluem incorporando elementos novos durante o enfrentamento com tradições rivais (MacIntyre 1981). Cameron, por sua vez, admite a existência do enfrentamento entre tradições e visões rivais; contudo, ao invés de aceitar mudanças, recomenda a defesa intransigente do antigo hipocratismo contra as inovações preconizadas pelos novos bioeticistas. Em Bioética, Leon Kass (ex-Presidente do Conselho Presidencial de Bioética nos Estados Unidos, o PCBE—The President's Council on Bioethics—órgão extinto pelo Presidente Barack Obama) é outro bioeticista eminete que reagiu de forma semelhante a mudanças na tradição—embora com menor intransigência. Porém, preservar certos valores tradicionais da prática médica em meio à incorporação dos princípios e valores liberais da nova bioética é certamente possível. Não há incompatibilidade prima facie entre a defesa dos princípios e valores implicados no respeito aos direitos individuais e aos direitos humanos e a preservação dos valores tradicionais que fazem da medicina uma profissão a serviço da proteção, promoção e reabilitação da saúde dos pacientes (Azevedo 2005).

Isso fica mais claro ao observarmos quais são as finalidades ou objetivos que atualmente orientam o exercício da medicina moderna. Há certo consenso hoje em dia em torno dos seguintes objetivos: promover, proteger ou recuperar a saúde, o que implica prevenir, tratar e, quando possível, curar doenças; aliviar os sintomas, a dor e o sofrimento físico e psíquico; evitar a morte prematura; e, por fim, esclarecer o paciente acerca de sua condição clínica, de seu prognóstico e das alternativas de tratamento disponíveis (Azevedo 2005, p. 49; Jonsen, Siegler e Winslade 1997). Esses objetivos são redescrições dos tradicionais objetivos hipocráticos, não obstante, atualizados, tendo-se em vista aquilo que melhor representa a prática atual da chamada "medicina tradicional" (mainstream medicine). 
Nenhum desses objetivos está em contradição com os princípios hipocráticos; nem estão em contradição com a ideia de que os pacientes são sujeitos de direitos, aptos a exigir dos médicos que sua liberda, bem como suas crenças e vontades sejam respeitadas (o chamado "princípio do respeito à pessoa humana" ou "princípio da autonomia" em bioética). Desses objetivos, porém, não se segue que os médicos estejam obrigados ou comprometidos a fazer tudo o que for tido como desejável por seus próprios pacientes. Em outras palavras, os pacientes não estão legal e moralmente aptos a exigir de seus médicos aquilo que julgam desejável. No nosso modelo, é resguardado aos médicos a prerrogativa de avaliar e prescrever a seus pacientes apenas o que julgam adequado dentro de certas restrições científicas. Por outro lado, médicos não estão autorizados a impor suas opiniões profissionais sobre os pacientes. Assim, prescrições médicas não podem ser executadas sem o consentimento destes. Isso talvez signifique que o que chamamos de "autonomia" do paciente diz respeito mais propriamente ao direito destes a serem informados e a recusar prescrições ou decisões médicas (ao direito a que não lhe sejam impostos exames, intervenções ou tratamentos) (Azevedo 2005). Mas e quanto aos objetivos melhoristas? Seriam eles também finalidades constitutivas da prática médica moderna? Se os médicos não estão obrigados a prescrever a seus pacientes aquilo que não julgam apropriado para atender aos objetivos da medicina, segue-se que práticas melhoristas somente poderiam ser implementadas caso passassem a figurar como objetivos da própria prática médica, algo que depende de consenso entre os próprios praticantes da atividade. Estariam também nesse caso os pacientes limitados a aceitar ou recusar aquilo que os médicos consideram um melhoramento?

Observe-se o seguinte: "promover, proteger e recuperar a saúde", talvez o principal objetivo da prática da medicina, é um objetivo que pressupõe um conceito de saúde algo restrito. Trata-se de um conceito médico de saúde. Em outras palavras, proteger a saúde em termos médicos é proteger um certo estado biológico descrito em termos médicos. Posso parecer estar sendo circular, mas o que estou afirmando é que "saúde" em termos médicos é um conceito imanente à medicina. Não é acidental que na prática comum da medicina, "saúde" seja compreendida como um estado de "ausência de doenças". Sabemos que há visões que divergem disso. Uma delas é a visão preconizada por muitos sanitaristas e pela área hoje conhecida como "saúde coletiva". Em saúde coletiva, "saúde" é compreendida como um estado "ampliado", que inclui não apenas a ausência de adoecimentos, mas a posse de capacidades e recursos capazes de garantir proteção contra o adoecimento, a morte prematura e a incapacidade humana. Porém, na prática clínica, as chamadas visões "ampliadas" do conceito de saúde não têm aplicação concreta. Pois, se o conceito orientador 
da prática clínica fosse esse, então os médicos estariam igualmente comprometidos a promover e realizar ações capazes de alterar a qualidade de vida de seus pacientes em âmbitos muito diversos para além de sua expertise. Isso porque ter saúde num sentido ampliado implica, por exemplo, ter renda mínima ou suficiente, escolaridade, saneamento básico e habitação digna. Além do mais, tais ações não são promovidas por ações ou serviços de saúde (ao menos não diretamente). Ora, promover renda mínima, escolaridade, condições adequadas de habitação e saneamento não é, como tal, um objetivo da prática médica, ainda que tais condições certamente contribuam para a promoção da saúde humana (em saúde coletiva chama-se a isso de promoção "primordial”). Faria, por outro lado, parte do escopo da prática clínica melhorar a qualidade de vida de uma pessoa para além daquilo que é possível alcançar por meio de ações preventivas, e por ações de cuidado ou terapêuticas médicas? Tenho defendido, de forma realista, que as ações médicas visam a objetivos restritos e específicos, e que os objetivos elencados pelas visões intituladas como "ampliadas" não são objetivos próprios da medicina. Assim, o que precisamos é de um conceito de saúde que descreva de forma mais adequada o que de fato corresponde à atividade clínica e mesmo à prática médica na área da saúde coletiva. No livro Bioética Fundamental, defendi o seguinte conceito: um indivíduo é saudável se seu risco de adoecimento e mortalidade é próximo ou igual ao melhor padrão de risco de adoecimento e mortalidade para indivíduos de seu grupo (Azevedo 2002, p. 99-100). Recentemente, busquei detalhar melhor esse conceito, afirmando que uma pessoa é saudável se ela não apresentar qualquer patologia crônica (ou condição patológica não transitória) que a submeta a um risco maior de morte ou adoecimento (físico ou mental), ou um maior risco de vir a tornar-se incapacitada (fisica ou mentalmente), do que o risco de morrer, adoecer ou de portar alguma incapacidade atribuído aos indivíduos de sua própria população grupo (ou classe de referência), porém, livres dessa patologia (Azevedo 2015). A ideia é mostrar que, em medicina ao menos, "saúde" é um conceito clínico-epidemiológico. Trata-se de um conceito operacional, isto é, de um parâmetro que permite aos médicos e profissionais de saúde estabelecer objeticos tanto em saúde coletiva como na prática clínica.

Note-se, porém, que esse conceito "saúde" é definido em termos estritamente médicos. Compare-o, porém, com outro conceito, igualmente "médico", o conhecido conceito "negativo" de que saúde é mera "ausência de doença" (Boorse 1975). Uma das falhas flagrantes do conceito negativo de saúde é que ele não é sensível o suficiente, pois ele inclui como não saudáveis indivíduos que se encontram com "boa saúde". Ao afastar da categoria "saúde" indistintamente indivíduos portadores de qualquer patologia, e sob quaisquer circunstâncias, acaba-se por excluir a possibilidade de que portadores de doenças agudas 
benignas e doentes crônicos adequadamente tratados possam ser considerados clinicamente saudáveis. Indivíduos com doenças diarreicas passageiras, resfriados ou mesmo lesões de pele (como verrugas virais) deveriam ser classificados como não saudáveis. Diabéticos e hipertensos, por exemplo, jamais poderiam ser considerados saudáveis, mesmo que tivessem sua doença plenamente controlada. Indivíduos míopes também deveriam segundo esse conceito ser considerados como não saudáveis (Murphy 1977). Ele também resulta em tomar como não saudáveis indivíduos que consideramos clinicamente hígidos, em vista das características de seu grupo etário. Por exemplo, todo homem com mais de 70 anos de idade terá Hiperplasia Benigna de Próstata e vários terão Câncer de Próstata; mulheres de mais de 60 anos têm alta probabilidade de serem portadoras de Osteoporose; todos os adultos com mais de 40 anos desenvolvem Presbiopia, etc. O conceito também resulta em afastar da categoria "saudável” qualquer portador de doença crônica tratável. Uma vez que uma doença crônica tenha se instalado, seus portadores jamais poderiam voltar a ser considerados hígidos-a menos que a doença fosse curada. No caso de doenças agudas, o resultado é paradoxal. Uma pessoa acometida de algum mal agudo deixaria de ser saudável (por alguns dias ou semanas) até o seu pleno restabelecimento. Ora, isso é contraintuitivo; os médicos costumam afirmar que seus pacientes são hígidos mesmo em situações de acomentimento agudo por doenças benignas (é justamente por isso que se curam sem tratamento algum). Também dizem que seus pacientes portadores de doenças crônicas são saudáveis caso encontrem-se ou tenham alcançado o melhor padrão possível de morbidade e mortalidade para a condição ou grupo de que fazem parte.

Assim, o conceito de que saúde equivale ao melhor padrão de risco de adoecimento e mortalidade para indivíduos de seu grupo pretende capturar de forma mais realista (e de forma mais precisa) o significado de "saúde" no âmbito da prática clínica sem incorrer nas falhas epistemológicas do conceito "negativo". O filósofo Christopher Boorse, no entanto, defendeu uma versão do conceito tradicional de saúde como ausência de doença, argumentando em favor da visão de que um indivíduo é saudável em termos "teóricos", "isto é, livre de doença”, na medida em que seu funcionamento conforma-se ao padrão (design) natural desse tipo de organismo (Boorse 1975, p. 57). Esse padrão (fisiológico) pode ser apresentado em termos "bioestatísticos" (daí a denominação de "teoria bioestatística", bioestatistical theory, ou BST, à teoria de Boorse). Todavia, há semelhanças de gênero evidentes entre o conceito clínico-epidemiológico de saúde e o conceito de Boorse; mas a vantagem do conceito que estou propondo é o de corrigir o conceito bioestatístico, adaptandoo a um ponto de vista epidemiológico. Ao fazer isso, admite-se também que "saúde" é uma 
condição "relativa", o que significa também retirar o conceito do domínio da fisiopatologia e submetê-lo a critérios clínicos. Por exemplo, em termos puramente fisiopatológicos, pessoas diabéticas são pessoas doentes. Entrentanto, em termos clínicos, faz sentido dizer que há diabéticos mais saudáveis que outros (embora seja verdadeiro que ser diabético representa mais riscos à saúde comparado a não sê-lo). Pessoas com deficiências são também pessoas "doentes", em um sentido puramente fisiopatológico; mas, mesmo que seja impossível reabilitá-las, é possível atenuar a deficiência a ponto de que o indivíduo aproxime-se de um padrão geral de adoecimento e mortalidade ótimo para seu grupo especial (e mesmo para o grupo geral). Suspeito que Boorse veria com cautela minha proposta, já que isso implica tomar a clínica como parâmetro (para Boorse, o parâmetro deve ser a patologia). Para Boorse, o problema é que a clínica é uma atividade prática, orientada, como tais a valores e objetivos-ela não é puramente "neutra", ou tão somente descritiva, como Boorse diz que é a fisiologia e a patologia.

Mas se saúde for um conceito vinculado a expectativas clínico-epidemiológicas padronizadas de adoecimento e mortalidade, então não faz sentido dizer de alguém que é mais saudável do que outro independemente desses padrões. Objetivos melhoristas, de todo modo, estão além do objetivo de promover, proteger e reabilitar a saúde humana. Curar um diabético certamente é torná-lo mais saudável do que apenas tratá-lo-mas aqui o parâmetro é o que é ótimo para certo grupo humano. Contudo, melhorar um ser humano é fazer mais do que torná-lo saudável. Por exemplo, ao tornar um atleta mais veloz do que Usain Bolt não estamos aproximando-o de qualquer parâmetro do que é ótimo para atletas ou para homens de sua idade. Não estamos fazendo dele uma pessoa mais saudável, pois nesse caso não estamos lidando com parâmetros biológicos historicamente selecionados. Estamos fazendo dele um atleta melhor, mais veloz do que era antes, embora tão saudável quanto são Bolt e os demais atletas em condições de competir com ele.

\section{O desafio do melhoramento humano às visões naturalistas}

Julian Savulescu tem sido um dos mais enfáticos defensores do que chama de "imperativo moral do melhoramento humano" (2004, 2005). Paralelamente, Savulescu também tem atacado as visões tradicionais sobre adoecimento e saúde em medicina (Ver: (http://blog.practicalethics.ox.ac.uk/2010/11/lethal-ethics-when-philosophical-distinctionskill/). Suas opiniões são polêmicas, mas seus argumentos são perspicazes. Savulescu representa um dos polos extremos nesse debate. O outro polo é representado pelos filósofos que temem que a proliferação de práticas melhoristas possa trazer danos a bens e valores que 
prezamos e consideramos essenciais à nossa identidade como seres dotados de humanidade. Alguns os chamam de bioconservadores.

Thomas Douglas apresenta do seguinte modo a tese bioconservadora. Segundo Douglas, os bioconservadores mantêm em comum a crença moral de que "mesmo que fosse tecnicamente possível e legalmente permissível o engajamento das pessoas no melhoramento biomédico, isso não seria moralmente permissível (Douglas 2008, p. 228). Mal qual seria a razão para essa objeção? Um dos argumentos bioconservadores mais difundidos apela a problemas de justiça distributiva (Buchanan et al 2000; Habermas 2001, 2010). Permitir o melhoramento humano, por meio de técnicas eugênicas, por exemplo, significaria reforçar e ampliar ainda mais a desigualdade humana, dado o acesso desigual a bens como rendas e riquezas. As novas desigualdades não conduziriam os menos avantajados a qualquer melhoria de sua condição anterior-contrariando um dos princípios recomendados por John Rawls, o chamado princípio da diferença (Rawls 1971). Mas um defensor do liberalismo político não parece ter argumentos de princípio contra a eugenia liberal. Desde que a distribuição do acesso à técnica seja reajustada de modo a não provocar mais desigualdades, práticas eugências não poderiam ser proibidas.

Michael Sandel $(2004,2007)$ também é apontado por Douglas como um dos expoentes do bioconservadorismo. Muito embora Sandel faça de fato parte desse grupo, seu argumento "bioconservador" é mais sutil. Para Sandel, práticas melhoristas podem conduzir à eliminação de certos traços essenciais à nossa identidade social como seres humanos. Ele alega, por exemplo, que a geração voluntária de diferenças entre as pessoas pode resultar na eliminação de uma das bases sobre as quais se erige o conceito liberal e moderno de responsabilidade: somos responsáveis pelas consequências das decisões que tomamos, mas não somos responsáveis pelas diferenças que temos em traços e dotes que não são resultado (intencional ou não intencional) de nossos próprios atos. Dotes genéticos são exemplos de características sobre as quais não somos responsáveis (não se pode obviamente culpar alguém por ter nascido com alguma deficiência). Esses dotes, aliás, são tomados como aspectos de legítima consideração em justiça distributiva justamente por não terem sido resultado de nossas ações, isto é, de atos, omissivos ou comissivos, cujas consequências pode-se atribuir responsabilidade. O problema principal, então, seria de que o melhoramento poderia conduzir-nos a uma situação de "hiperagência". Como o melhoramento (genético, principalmente) estaríamos em condições de eliminar esses infortúnios. Nosso futuro como seres vivos se tornaria objeto completo de nossos desejos no presente. E se técnicas 
melhoristas fossem disponíveis universalmente, nada mais seria acidental. Tudo estaria sob o domínio de nossa decisão. Seria isso desejável?

Sandel acredita que não, pois com isso perderíamos o que chama senso de graça (the sense of giveness), algo que poderia afetar radicalmente nossas atitudes de solidariedade e humildade perante a vida, bem como de responsabilidade frente aos demais. Segundo Sandel, a solidariedade humana existe justamente porque reconhecemos que há infortúnios com respeito aos quais é injusto atribuir a qualquer pessoa responsabilidade. $\mathrm{O}$ fato de haver diferenças fortuitas é o que nos aproximaria e geraria entre nós um senso de comunidade e pertencimento. $\mathrm{O}$ argumento, no entanto, não me parece convincente. $\mathrm{O}$ temor de que práticas melhoristas, especialmente as eugênicas, eliminariam nosso senso de solidariedade e de responsabilidade individual por nossas decisões parece-me infundado e exagerado. Para entender isso não é preciso fazer especulações mirabolantes sobre um futuro de controle tecnológico absoluto sobre a vida (como por vezes as discussões sobre o emprego futuro de novas biotecnologias sugerem). Pensemos no caso do uso de estimulantes no esporte. Podemos ser contra seu uso, mas não porque sem emprego elimina o mérito e a responsabilidade. Se todos os corredores de uma maratona fossem igualados em condições padronizadas, mesmo que isso fosse garantido pela permissão do uso de substâncias hoje ilícitas, sendo elas não prejudiciais à saúde, por que pensaríamos que o vencedor não teria mérito pela vitória? Sandel acredita que a solidariedade depende de que existam infortúnios. Mas há também solidariedade e consolo simpático diante de insucessos. Além disso, podemos e geralmente somos solidários diante de erros e males sobre os quais as pessoas têm alguma responsabilidade (dependentes químicos são em algum grau responsáveis por sua doença; mesmo assim somos solidários com aqueles que desejam ver-se livres dessa mazela). Contudo, mesmo que admitamos que Sandel tem um argumento interessante, sua posição não é incompatível com a aceitação controlada e limitada de práticas melhoristas.

Savulescu, contudo, é um consequencialista em ética e um não-descritivista sobre a natureza da normatividade. Assim como os bioconservadores, Savulescu vê a ética como instrumento em favor de nossas melhores aspirações (em seu caso, aspirações seguramente não-conservadoras). Mas, diferentemente dos bioconservadores (especialmente dos teólogos), Savulescu não pensa o problema tendo em vista qualquer concepção metafísica sobre a natureza humana. Seu foco está em perguntar-se se seria ou não racional para alguém desejar melhorar seus dotes naturais ou genéticos. Ainda assim, Savulescu parte de um imperativo duvidoso: a ideia de que os indivíduos têm um "dever moral" de agir racionalmente visando sempre os melhores resultados (Persson \& Savulescu 2008, 2012). De minha parte, suspeito 
que Savulescu faça um uso apenas retórico dos termos "obrigação" e "imperativo". Pois, se tomássemos a alegação de Savulescu a sério deveríamos também acreditar que toda pessoa que deixasse de buscar aprimorar-se estaria cometendo um erro indesculpável de natureza moral. Ora, ninguém está obrigado, nem mesmo "moralmente", a melhorar a si ou aos outros. Se isso fosse verdadeiro, deveríamos estar autorizados a forçar as pessoas a serem melhoradas contra sua vontade. Levando a sério a ideia de obrigação (ou seja, de que toda obrigação genuína implica a permissão de alguma forma de coerção em favor da parte prejudicada, pois, se obrigações nunca pudessem ser corrigidas, estaríamos diante de uma grave injustiça contra aqueles cujos direitos foram violados), conclui-se que melhoramentos jamais poderiam ser forçados, a menos que alguém tivesse o direito a que outra pessoa fosse melhorada em seu benefício. Ora, obrigação e permissibilidade são conceitos normativos distintos; mesmo que o melhoramento humano seja em certos casos recomendável, disso ainda não se segue que seja estrita e literalmente obrigatório.

\section{Conclusão: ampliando os objetivos da medicina}

Não há, enfim, impedimentos de princípio a que o melhoramento humano passe a figurar como um dos objetivos da prática médica. Isso já ocorre de certa maneira em vários contextos, como é o caso da medicina estética. Contudo, se o melhoramento humano for incluído como um objetivo da medicina, ainda assim não faz sentido equipará-lo ao objetivo de promover e proteger a saúde das pessoas. Médicos não têm apenas a permissão de promover a saúde de seus pacientes; trata-se de um dever estrito, a saber, o dever de agir visando sempre promover e proteger a saúde de seus pacientes, sob pena de responsabilidade moral, além de civil e profissional. É por isso que dizemos dos médicos que oferecem aos doentes alternativas comprovadamente piores sem justificativa plausível que agem de forma negligente. Ora, mesmo que a medicina incorpore práticas melhoristas, não faz sentido dizer de um médico que não as ofereça a seu paciente que tenha agido de forma negligente.

Considere a seguinte situação. Suponhamos que num futuro próximo, médicos possam ser autorizados legalmente a prescrever medicamentos capazes de melhorar a performance dos atletas. Nesse caso, se o atleta não obtiver a performance desejada, faria sentido dizer que o médico cometeu um erro e que deve por isso ser responsabilizado? Se um médico deixar de fornecer ao paciente o melhor tratamento disponível para sua doença, certamente faz sentido acusá-lo de negligência. Mas isso não se estende às práticas melhoristas. Alguns diriam que isso equivaleria a tomar os objetivos melhoristas como compromissos de fins e não de meios.

Barbarói, Santa Cruz do Sul, Edição Especial n.44, p.<290-303>, jul./dez. 2015 
Ora, se objetivos melhoristas não podem ser tomados como compromissos de "fins", que tipo de objetivo seria esse? Trata-se de uma situação aparentemente paradoxal que merece ser estudada. $\mathrm{O}$ descortinamento desse aparente paradoxo poderá iluminar nossa compreensão sobre a natureza da responsabilidade médica e sobre os limites da incorporação de práticas melhoristas no seio da prática clínica. É razoável que devamos incorporar ao seio da medicina não apenas ações de cuidado e tratamento de doenças, mas também ações clínicas de melhoramento. Sou pessoalmente a favor disso, pois não vejo motivos categóricos para que proibamos melhoramentos por princípio. Porém, antes que isso venha a ocorrer de forma sistemática, é recomendável que avaliemos (médicos e sociedade) de que modo inseri-las sem descaracterizar os fins essenciais da profissão, e, principalmente, sem reduzir a medicina a uma prática comum de balcão, ainda executada por técnicos dispostos a oferecer opções a qualquer tipo de desejo ou capricho pessoal sem constrangimentos.

\section{Referências}

Azevedo, M.A.O. A profissão sob risco. In: Agosto, Flávio Moura de; Peixoto, Rui \& Bordin, Ronaldo (Org.). Riscos da prática médica. Porto Alegre: Dacasa, 1998, p. 79-92.

. Bioética fundamental. Porto Alegre: Tomo Editorial, 2002.

Decisões clínicas eticamente orientadas. Revista AMRIGS, 49 (1), 2005: 44-

51. Acesso em: http://www.amrigs.com.br/revista/49-01/cremers4901.pdf.

. Health as a clinic-epidemiological concept. Journal of Evaluation in Clinical

Practice 21, 2015: 365-373 (doi:10.1111/jep.12279).

Battleday, R.M. \& A.-K., Brem. Modafinil forcognitiveneuroenhancement in healthynonsleep-deprived subjects: A systematic review. European Neuropsychopharmacology 25 (11) 2015: 1865-81. doi:10.1016/j.euroneuro.2015.07.028

Boorse, C. Health as theoretical concept. Philosophy of Science 44 (4), 1977: 542-573.

On the distinction between disease and illness. Philosophy \& Public Affairs 5, 1975: 49-68.

Buchanan, A.; Brock, D.; Daniels, N. \& Wikler, D. From chance to choice: genetics and justice. Cambridge University Press, 2000.

Cameron, Nigel M. de S. The new medicine: life and death after Hippocrates. Crossway Books, 1991.

Douglas, Thomas. Moral enhancement. Journal of Applied Philosophy 25 (3), 2008: 228-245. 
Elliot, C. Better than well: American medicine meets the American dream. W. W. Norton, 2003.

Foot, P. Natural goodness. Oxford: Clarendon Press, 2001.

Freidson, E. Profession of medicine. The University of Chicago University Press, 1988. Professionalism, the third logic. The University of Chicago Press, 2001.

Habermas, J. O futuro da natureza humana. São Paulo: Martins Fontes, 2010 (tradução de Die Zukunft der menschlichen natur. Auf dem weg zu einer liberalen Eugenik? Frankfurt am Main: Suhrkamp Verlag, 2001).

Jonsen, A. R, Siegler, M. \& Winslade, W.J. Clinical ethics. 4th edition. McGraw-Hill Companies, 1997.

Kumar, R. Approved and investigational uses of modafinil: an evidence-based review. Drugs 68 (13), 2008: 1803-1839. http://dx.doi.org/10.2165/ 00003495-200868130-00003.

MacIntyre, A. After virtue. Duckworth, 1981.

Marken, Patricia A \& Munro, J. Stuart. Selecting a Selective Serotonin Reuptake Inhibitor. Clinically Important Distinguishing Features. Primary Care Companion of the Journal of Clinical Psychiatry 2 (6) 2000: 205-210.

Murphy, E. A. The logic of medicine. 2nd Edition. The Johns Hopkins University Press, 1977.

Persson, I. \& Savulescu, J. The perils of cognitive enhancement and the urgent imperative to enhance the moral character of humanity. Journal of Applied Philosophy 25 (3), 2008: 162167.

Persson, I. \& Savulescu, J. Unfit to the future. The need ofr moral enhancement. Oxford University Press, 2012.

Rapoport, J.L.; Buchsbaum, M.S.; Weingartner, H.; Zahn, T.P.; Ludlow, C.; Mikkelsen, E.J. Dextroamphetamine. Its cognitive and behavioral effects in normal and hyperactive boys and normal men. Archives of General Psychiatry 37, 1980: 933-943.

Rapoport, J.L. \& Inoff-Germain, G. Responses to methylphenidate in attentiondeficit/hyperactivity disorder and normal children. Update 2002. J Atten Disord 6, 2002: S57S60.

Rawls, J. A theory of Justice. Harvard University Press, 1971.

Sandel, M. J. The case against perfection. The Atlantic Monthly 293 (3), 2004: 50-65.

The case against perfection. Ethics in the age of genetic engineering. The Belnak Press of Harvard University Press, 2007. 
Savulescu, J. Why I believe parents are morally obliged to genetically modify their children. Times Higher Education Supplement, 5, 2004.

- New breeds of humans: the moral obligation to enhance. Reproductive BioMedicine Online, 10 (1), 2005: 36-39.

Scheffler, R.M.; Brown, T.T.; Fulton, B.D.; Hinshaw, S.P,; Levine, P. \& Stone, S. Positive association between attention-deficit/ hyperactivity disorder medication use and academic achievement during elementary school. Pediatrics 123, 2009: 1273-1279.

Spencer, R.C; Devilbiss, D.M.; Berridge, C.W. The Cognition-Enhancing Effects of Psychostimulants Involve Direct Action in the Prefrontal Cortex. Biological Psychiatry 77 (11), 2015: 940-950.

\section{Sobre o autor:}

Marco Antonio Azevedo é Médico e Professor de Filosofia da Unisinos. 